2014

A workflow for bacterial metabolic fingerprinting and lipid profiling: application to Ciprofloxacin challenged Escherichia coli

\author{
Allwood, JW \\ http://hdl.handle.net/10026.1/11126
}

10.1007/s11306-014-0674-6

Metabolomics

Springer US

All content in PEARL is protected by copyright law. Author manuscripts are made available in accordance with publisher policies. Please cite only the published version using the details provided on the item record or document. In the absence of an open licence (e.g. Creative Commons), permissions for further reuse of content should be sought from the publisher or author. 


\title{
A workflow for bacterial metabolic fingerprinting and lipid profiling: application to Ciprofloxacin challenged Escherichia coli
}

\author{
J. William Allwood · Haitham AlRabiah • \\ Elon Correa · Andrew Vaughan · Yun Xu • \\ Mathew Upton · Royston Goodacre
}

Received: 24 March 2014 / Accepted: 8 May 2014/Published online: 24 May 2014

(c) Springer Science+Business Media New York 2014

\begin{abstract}
The field of lipidomics focuses upon the nontargeted analysis of lipid composition, the process of which follows similar routines to those applied in conventional metabolic profiling, however lipidomics differs with respect to the sample preparation steps and chosen analytical platform applied to the sample analysis. Conventionally, lipidomics has applied analytical techniques such as direct infusion mass spectrometry and more recently reverse phase liquid chromatography-mass spectrometry, for the detection of mono-, di-, and tri-acyl glycerols, phospholipids, and other complex lipophilic species such as sterols. The field is rapidly expanding, especially with respect to the clinical sciences where it is known that changes of lipid composition, especially phospholipids, are commonly associated with many disease processes. As a
\end{abstract}

J. William Allwood and Haitham AlRabiah have contributed equally to this publication.

Electronic supplementary material The online version of this article (doi:10.1007/s11306-014-0674-6) contains supplementary material, which is available to authorized users.

J. W. Allwood $(\bowtie) \cdot$ H. AlRabiah · E. Correa · A. Vaughan .

Y. Xu $\cdot$ R. Goodacre

Manchester Institute of Biotechnology, School of Chemistry,

University of Manchester, 131 Princess Street,

Manchester M1 7DN, UK

e-mail: j.w.allwood@bham.ac.uk

J. W. Allwood

School of Biosciences, University of Birmingham, Edgbaston, Birmingham B15 2TT, UK

M. Upton

Microbiology and Virology Unit, School of Translational

Medicine, University of Manchester, Stopford Building, Oxford

Road, Manchester M13 9PL, UK proof of principle study, a small number of Escherichia coli isolates were selected on the basis of their sensitivity to a second generation fluoroquinolone antibiotic, known as Ciprofloxacin (E. coli isolates 161 and 171, non-ST131 isolates, which are resistant and sensitive respectively: E. coli isolates 160 and 173, ST131 sequence isolates which are resistant and susceptible respectively). It has been proposed that Ciprofloxacin may be a surface active drug that interacts at the surface-water interface of the phospholipid bi-layer where the head groups reside. Further, antibiotic resistance through intracellular exclusion is known to result in remodelling of the phospholipid membrane. Therefore, to study the effects of Ciprofloxacin on both susceptible and resistant bacterial strains, lipid profiling would present an informative approach. Control and antibiotic challenged cultures for each of the isolates were compared for changes in metabolite and lipid composition as detected by FT-IR spectroscopy and RP-UHPLC-MS, and appraised with a variety of chemometric data analysis approaches. The developed bacterial lipidomics workflow was deemed to be highly reproducible (with respect to the

\footnotetext{
M. Upton

School of Biomedical and Healthcare Sciences, Plymouth

University, Drake Circus, Plymouth PL4 8AA, UK

R. Goodacre

Manchester Centre for Integrative Systems Biology, Manchester Institute of Biotechnology, School of Chemistry, University of Manchester, 131 Princess Street, Manchester M1 7DN, UK
} 
employed technical and analytical routines) and led to the detection of a large array of lipid classes as well as highlighting a range of significant lipid alterations that differed in regulation between susceptible and resistant $E$. coli isolates.

Keywords Lipidomics - Escherichia coli C Ciprofloxacin hydrochloride - Antibiotic-resistance $\cdot$ Fourier transform infrared spectroscopy $\cdot$ Liquid chromatography-mass spectrometry

\section{Introduction}

The popularity of metabolomics and the closely related discipline of lipidomics has risen greatly over the past decade, across an ever growing range of disciplines and applications, including, gene function analysis (Fiehn et al. 2000; Roessner et al. 2001), environmental perturbation (Kaplan et al. 2004; Allwood et al. 2006, 2010), diseases of clinical significance (Kolak et al. 2007; Mattila et al. 2008; Orešič et al. 2008; Sreekumar et al. 2009; Kenny et al. 2010), and to a large number of organisms including plants (Lisec et al. 2006; De Vos et al. 2007), fruits, vegetables and grains (Allwood et al. 2009; Biais et al. 2009; Tikunov et al. 2010), microbes (Koek et al. 2006; Winder et al. 2006, 2008; Van Der Werf et al. 2008; Lowe et al. 2010), and mammals (Griffin and Kauppinen 2007; Sreekumar et al. 2009; Kenny et al. 2010; Velagapudi et al. 2010; Dunn et al. 2007, 2008, 2011). The metabolome and lipidome perhaps represents the ultimate phenotype of cells, controlled by gene expression and the modulation of protein function, which in turn are controlled by the environment and genome mutation (Fiehn 2002; Saito and Matsuda 2010). The field of lipidomics focuses upon the non-targeted analysis of lipid composition, the process of data collection and statistical analysis follows very similar routines to those applied in conventional metabolic profiling, however lipidomics differs with respect to the sample preparation steps and chosen analytical platform applied to the sample analysis. Conventionally, lipidomics has applied analytical techniques such as direct infusion mass spectrometry (DIMS; Goodacre et al. 2002; Han and Gross 2005; Allwood et al. 2006) and less commonly reverse phase liquid chromatography-mass spectrometry (RP-LC-MS; Kolak et al. 2007; Mattila et al. 2008; Wedge et al. 2011), for the detection of mono-, di-, and tri-acyl glycerols, phospholipids, and other complex lipophilic species such as sterols and ceramides.

Given the influence and importance of microbes upon many areas of biological, clinical and environmental research, they are indeed a very significant target for metabolomics and lipidomics. Many studies have focused upon the polar central metabolism of microbes (Koek et al. 2006; Winder et al. 2008; MacKenzie et al. 2008), including applications in several systematic studies to increase our level of understanding on the control of central metabolism at the levels of the transcriptome, proteome and metabolome, with such studies typically focusing upon model organisms such as Escherichia coli K-12 (Mori 2004) and Saccharomyces cerevisiae (Castrillo et al. 2007; Herrgård et al. 2008). Further to E. coli being a commonly employed model species (Mori 2004; Riley et al. 2006), it is also of great significance as an enteric pathogen (Kaper et al. 2004), requiring antibiotic treatments in mammals to clear the gastric infection and alleviate symptoms of gastroenteritis. Understanding the actions of antibiotics, which are frequently poorly described, at the levels of the metabolome and lipidome using model bacteria such as E. coli is of importance to elucidate the mechanisms of action of antibiotics in current use and those under development. Antibiotics are commonly targeted to specific proteins, for example, fluoroquinolones such as Ciprofloxacin, are targeted to the bacterial DNA topoisomerase II (DNA gyrase) and DNA topoisomerase IV enzymes which function to relax supercoiled DNA and perform important processes in partitioning chromosomal DNA respectively (Greenwood 2000). Therefore, to study the effects of Ciprofloxacin on the metabolism of bacteria, especially those that are sensitive to the antibiotic, it would be logical to employ an analytical technique that measures the central metabolome (Koek et al. 2006; Winder et al. 2008). Such an approach would be capable of detecting changes in nucleotide metabolism and how they impact upon other areas of primary metabolism, potentially revealing direct consequential effects on metabolism due to the antibiotic mode of action. However, with the ever growing problem of bacterial resistance to antibiotics and the emergence of bacteria with resistance to multiple antibiotics (Tomasz 1994; Wiener et al. 1999; Sáenz et al. 2004), for example Methicillin-resistant Staphylococcus aureus (MRSA; Diederen and Kluytmans 2006), it is perhaps more important to focus studies on bacterial modes of resistance. Since bacterial resistance to antibiotics is commonly dependent on the ability of the bacteria to exclude the antibiotic from the cell by membrane remodelling via aminoacylation or cell wall modification by peptidoglycan cross-linking (RajBhandary and Söll 2008; Roy and Ibba 2008), or alternatively by removal of the antibiotic from the cytoplasm to the extracellular matrix through energy dependent membrane export (Poole et al. 1993; Okusu et al. 1996), it may be of great relevance to alternatively analyse the lipidome of bacteria.

Much research has been previously undertaken on the optimisation of bacterial growth and metabolic quenching, as well as the development of suitable GC-MS 
methodologies for the analysis of central metabolism in E. coli as well as other bacterial species and yeasts (Winder et al. 2008; MacKenzie et al. 2008). Therefore, the principle aim of this investigation was to establish and validate a method for FT-IR fingerprinting and RP UHPLC-MS profiling of lipids in antibiotic challenged $E$. coli, taking into consideration sample extraction and analysis steps. FT-IR spectroscopy is a constantly developing analytical technique often used routinely as a method in bacteriology being applied to species level identification and discrimination, as well as a tool to follow phenotypic responses to abiotic and biotic perturbation (Winder et al. 2006; Preisner et al. 2007; Wehlri et al. 2013). RP LC-MS employing C18 column chemistries is the standard approach applied to the analysis of free and membrane lipids in the field of lipidomics. Therefore both FT-IR and RP UHPLC-MS were deemed appropriate analytical tools to apply to the study of changes in lipid quantity and composition in response to antibiotic challenge in bacteria.

As a proof of principle study, a small number of E. coli isolates were selected on the basis of their sensitivity to Ciprofloxacin. The isolates chosen are clinically relevant, being recently recovered from cases of urinary tract infection, and included strains from the globally disseminated ST131 clone (Lau et al., 2008). E. coli isolates 160 and 173, of the ST131 clone, which are classified as resistant and sensitive respectively, as well as E. coli isolates 161 and 171, non-ST131 strains, which are classified as resistant and intermediate resistant respectively, were selected to provide a range of $E$. coli responses to challenge with Ciprofloxacin. It has been proposed that Ciprofloxacin may be a surface active drug that interacts at the surfacewater interface of the phospholipid bi-layer where the head groups reside (Merino et al. 2003). Further, since antibiotic resistance through intracellular exclusion is known to result in remodelling of the phospholipid membrane, the developed FT-IR fingerprinting and RP-UHPLC-MS lipid profiling approach was deemed to provide an informative method to study the effects of Ciprofloxacin on both susceptible and resistant bacterial responses.

\section{Materials and methods}

\subsection{Chemicals}

All chemicals used were of analytical reagent or a higher purity grade. All reference standards were of $99 \%$ minimum purity. All materials were purchased from SigmaAldrich (Gillingham, UK) unless otherwise stated. Ciprofloxacin hydrochloride was obtained from Discovery fine chemicals (Dorset, UK), a stock solution was prepared by dissolving $100 \mathrm{mg}$ in $50 \mathrm{ml}$ of sterile distilled water, further dilutions were also made with sterile distilled water. HPLC grade methanol, chloroform and water were purchased from Sigma-Aldrich (Gillingham, UK). Formic acid (BDH Aristar 1) was purchased from VWR International (East Grinstead, UK).

\subsection{Escherichia coli isolates}

Four clinical E. coli isolates were donated by Dr. Mathew Upton from the Manchester Royal Infirmary. The isolates were selected on the basis of sequence type and sensitivity towards quinolones. A sensitive isolate (isolate 173) and a resistant isolate (isolate 160) of the ST131 clone, as well as two isolates that were resistant and partially-resistant to quinolones, isolates 161 and 171 respectively (non-ST131 strains), were selected for the lipidomic analysis.

\subsection{Preparation of Escherichia coli cultures, their} antibiotic challenge and determination of minimal inhibitory concentration (MIC)

Preparation of E. coli inoculates, their culture, challenge with Ciprofloxacin and determination of MIC are described in full detail within the Supplementary methods (Sects. 1.1-1.3) and are also presented within Fig. 1. For both the determination of MIC and also generation of samples for metabolic fingerprinting and profiling, all cultures were generated on a single day, with a single batch of culture media and were incubated in a common orbital shaker. In the case of larger experiments where sample numbers demand the generation of cultures in multiple batches (across days and even weeks), it is recommended that a minimum of one sample class is maintained within all culture batches, therefore providing a measure of variance associated with the different days/batches of culture generation. Further, by generating inoculums and culture media as a single lot for all experimental batches, as well as preparing a single source of both quenching and extraction solvents, biological and technical variation between the experimental batches will be minimised. In total 24 cultures were generated ( 4 E. coli strains $\times 2$ treatments (control and antibiotic challenged) $\times 3$ biological replicates). The experiment was designed to test technical (sample quenching) and analytical reproducibility, with having only a minimal number of biological replicates generated within a single culture batch, the experimental design does not permit a measure of the biological reproducibility of the employed methods.

2.4 Escherichia coli sample collection and quenching for metabolic fingerprinting and lipid profiling

The sample collection and quenching of metabolism methods were adapted from the procedures developed by 


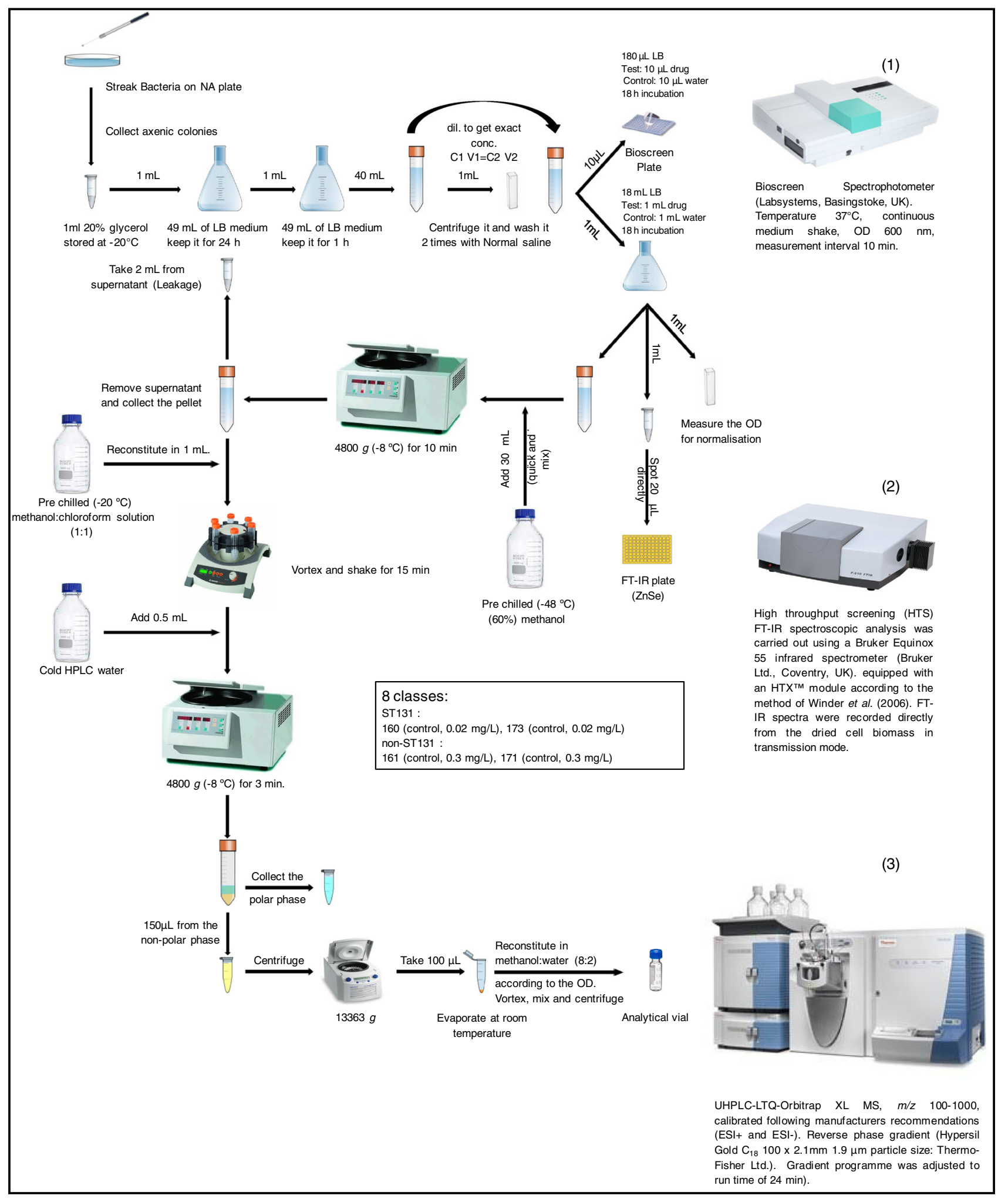

Fig. 1 Schematic of sample generation including (1) bioscreen analysis to determine the MIC of ciprofloxacin and produce the growth curves of pathogenic E. coli. (2) FT-IR analysis of samples.
(3) LC-MS analysis of samples after quenching with cold $\left(-48{ }^{\circ} \mathrm{C}\right)$ methanol and extraction with $(1: 1)$ methanol:chloroform 
Winder et al. (2008) (Fig. 1), a full description of which is available in the Supplementary methods Sect. 1.4. All bacterial cultures were collected on a single day. Due to limitations in sample handling capacity, quenching was performed upon randomised batches of six samples at a time and the procedure requires approximately $20 \mathrm{~min}$ per a batch, therefore the antibiotic treatment of each randomised batch was staggered across 20 min intervals, thus maintaining that each culture is challenged by the antibiotic for precisely $18 \mathrm{~h}$. By quenching the samples across randomised batches an indication of the procedures technical reproducibility can be obtained.

\subsection{Fourier transform infrared (FT-IR) spectroscopy}

High throughput screening (HTS) FT-IR spectroscopic analysis was carried out using a Bruker Equinox 55 infrared spectrometer (Bruker Ltd., Coventry, UK) equipped with an $\mathrm{HTX}^{\mathrm{TM}}$ module according to the method of Winder et al. (2006). Preparation of samples, FT-IR sample plates and acquisition of spectra are fully described in the Supplementary methods Sect. 1.5. Each biological sample was repeat spotted on the FT-IR plate and analysed in triplicate to provide an indication of analytical variance.

\subsection{Sample extraction for UHPLC-MS lipid profiling}

The bacterial samples were extracted applying methanol:chloroform (1:1), followed by addition of water so that polar and non-polar metabolites could be isolated via phase separation. The method is fully described in the Supplementary methods Sect. 1.6 and was adapted from the methods described in Winder et al. (2008), which were originally conceived by Bligh and Dyer (1959). The extraction method leads to the capture of both free and membrane lipids within the chloroform phase of the extract, all of which are amenable to separation and detection by RP-UHPLC-MS approaches.

\subsection{UHPLC-MS analysis}

All samples were analysed on the Accela UHPLC system (Thermo-Fisher Ltd. Hemel Hempsted, UK) coupled to an electrospray LTQ-Orbitrap XL hybrid mass spectrometry system (ThermoFisher, Bremen, Germany) adapting the methods previously described by Wedge et al. (2011) and provided in full detail in the Supplementary methods Sect. 1.7.

\subsection{Processing of raw UHPLC-MS profiles and lipid identification}

The UHPLC-MS raw data profiles were deconvolved using the freely available XCMS software (http://masspec. scripps.edu/xcms/xcms.php), the data were quality assured as described in (Dunn et al. 2008; Wedge et al. 2011) and normalised by peak sum for each sample (i.e. TIC normalisation). The putative identification of lipid features was performed applying the PUTMEDID-LCMS set of workflows (Brown et al. 2011), and a table of the putative lipid assignments is available in the supplementary information (Table S2). Detailed descriptions of deconvolution, quality assurance and putative lipid identification are provided in Supplementary methods Sect. 1.8.

\subsection{Statistical analyses}

Statistical analyses were performed applying the multivariate method of principal component-discriminant function analysis (PC-DFA). PC-DFA scores plots were employed for data visualisation and loadings plots to indicate the most significant metabolic features. Further feature selection was performed on the UHPLC-MS data for each individual bacterial isolate, comparing the response between the antibiotic treated and non-treated controls. First, a multivariate method known as partial least squares (PLS) regression was applied, this resulted in a filtered list of the most significant features ordered by the magnitude of their PLS regression coefficients from largest to smallest. Secondly, a univariate approach, known as ANOVA $(p<0.05)$, was applied to the significant PLS regression coefficient lists. Finally, a list of the 50 most significant features, ordered according to the magnitude of their PLS regression coefficients (from high to low), which also had an ANOVA $p$ value of $<0.05$, was produced for each bacterial strain. Full descriptions of the statistical methods are provided in Supplementary methods Sects. 1.9 and 1.10 .

\section{Results and discussion}

\subsection{Determination of the Ciprofloxacin minimal} inhibitory concentration (MIC)

So that the lipidomics approach under development did not lead to the detection of lipid changes associated with the death of bacterial cells as opposed to detecting lipid alterations that may contribute to the ability of the bacteria to exclude and remove the antibiotic, thus mediating antibiotic resistance, it was first necessary to establish the MIC of each bacterial isolate when cultured in Lysogeny Broth (LB) and challenged by Ciprofloxacin. Each of the four E. coli clinical isolates were cultured in LB media and challenged with Ciprofloxacin solutions ranging from 0.0025 to $100 \mathrm{mg} / \mathrm{l}$ in concentration. Bacterial growth curves were generated by measuring the OD $600 \mathrm{~nm}$ across an $18 \mathrm{~h}$ growth period 

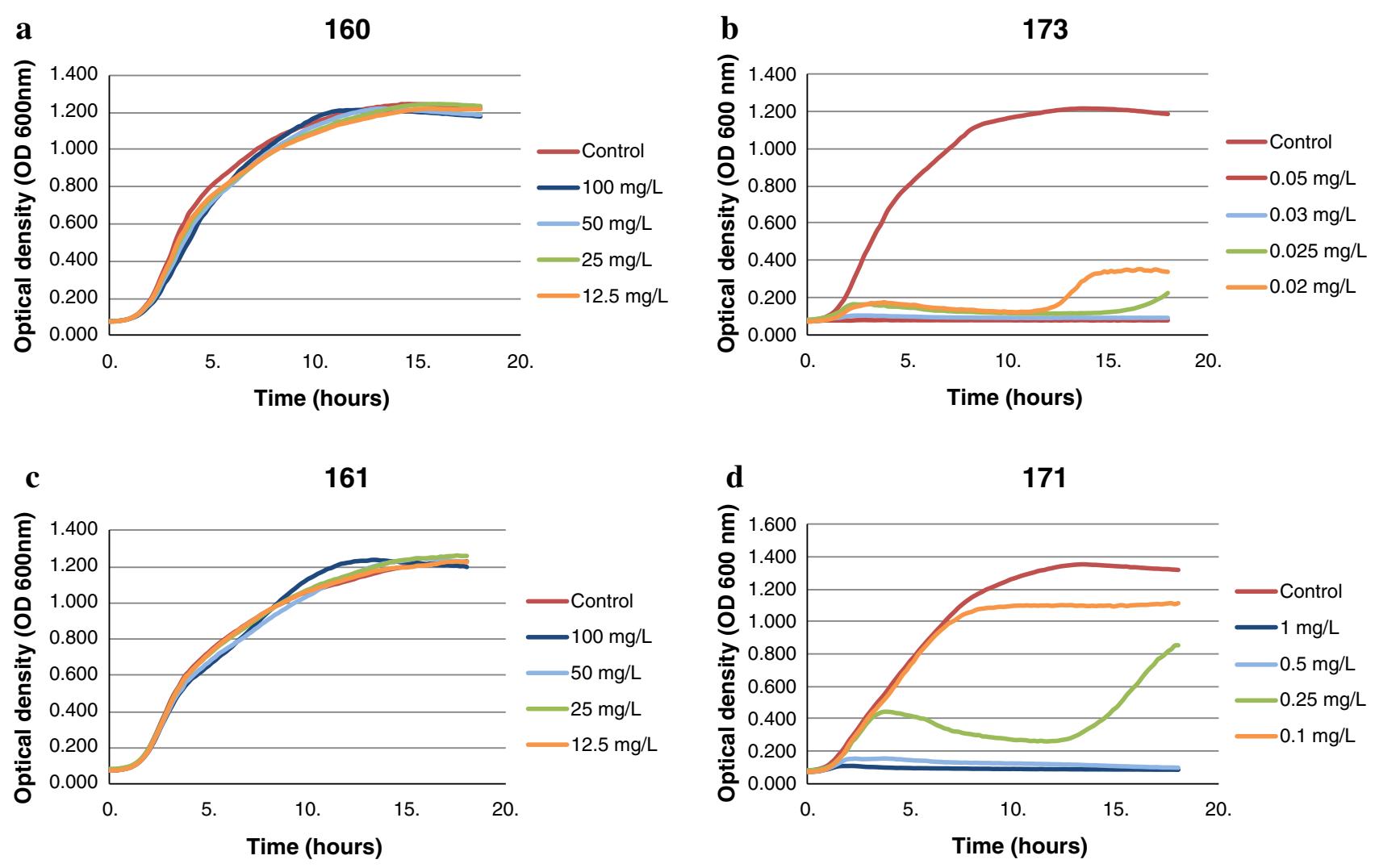

e

\begin{tabular}{|c|c|c|c|c|}
\hline Isolate number & Sequence type & Quinolone phenotype & $\begin{array}{c}\text { MIC range determination } \\
(\mathbf{m g} / \mathbf{L}) *\end{array}$ & $\begin{array}{c}\text { Selected dose to } \\
\text { challenge the } \\
\text { bacteria (mg/L) }\end{array}$ \\
\hline 160 & ST131 & Fully resistant & No effect & 0.02 \\
\hline 173 & ST131 & Sensitive & $0.03-0.02$ & 0.02 \\
\hline 161 & non-ST131 & Fully resistant & No effect & 0.3 \\
\hline 171 & non-ST131 & Intermediate resistant & $0.5-0.25$ & 0.3 \\
\hline
\end{tabular}

* Ciprofloxacin hydrochloride highest dose used is $100 \mathrm{mg} / \mathrm{L}$.

Fig. 2 OD 600 growth curves from 0 to $18 \mathrm{~h}$ post antibiotic challenge to indicate minimal inhibitory concentrations (MIC) of Ciprofloxacin hydrochloride against Escherichia coli ST131 strains

(Fig. 2). It was determined that the $E$. coli isolates 161 (nonST131) and 160 (ST131), regarded as fully resistant, were not inhibited in growth even at Ciprofloxacin concentrations as high as $100 \mathrm{mg} / \mathrm{l}$. For the intermediate-resistant E. coli isolate 171 (non-ST131) the Ciprofloxacin MIC was determined to fall between 0.25 and $0.5 \mathrm{mg} / \mathrm{l}$. Whereas for the sensitive E. coli isolate 173 (ST131) the Ciprofloxacin MIC was determined to fall between 0.02 and $0.03 \mathrm{mg} / \mathrm{l}$. Based upon these results, the concentrations of antibiotic were selected for the challenge of bacteria for sample generation for metabolic fingerprinting and lipid profiling. For the a 160 (resistant) and b 173 (sensitive) and non-ST131 strains c 161 (resistant) and d 171 (intermediate resistance), e table of predicted MIC (mg/l Ciprofloxacin)

E. coli ST131 isolates 160 and 173, in order to permit direct comparison of metabolic responses, a common antibiotic concentration of $0.02 \mathrm{mg} / \mathrm{l}$ was selected based upon it being the MIC of the sensitive isolate 173. For the non-ST131 isolates 161 and 171, again in order to permit direct comparison of metabolic responses, a common antibiotic concentration of $0.3 \mathrm{mg} / \mathrm{l}$ was selected based upon it being intermediate to the MIC of $0.25-0.5 \mathrm{mg} / \mathrm{l}$ of isolate 171 , and as being of a great enough concentration to lead to an inhibitory effect upon growth without resulting in total bacterial death (Fig. 2). 

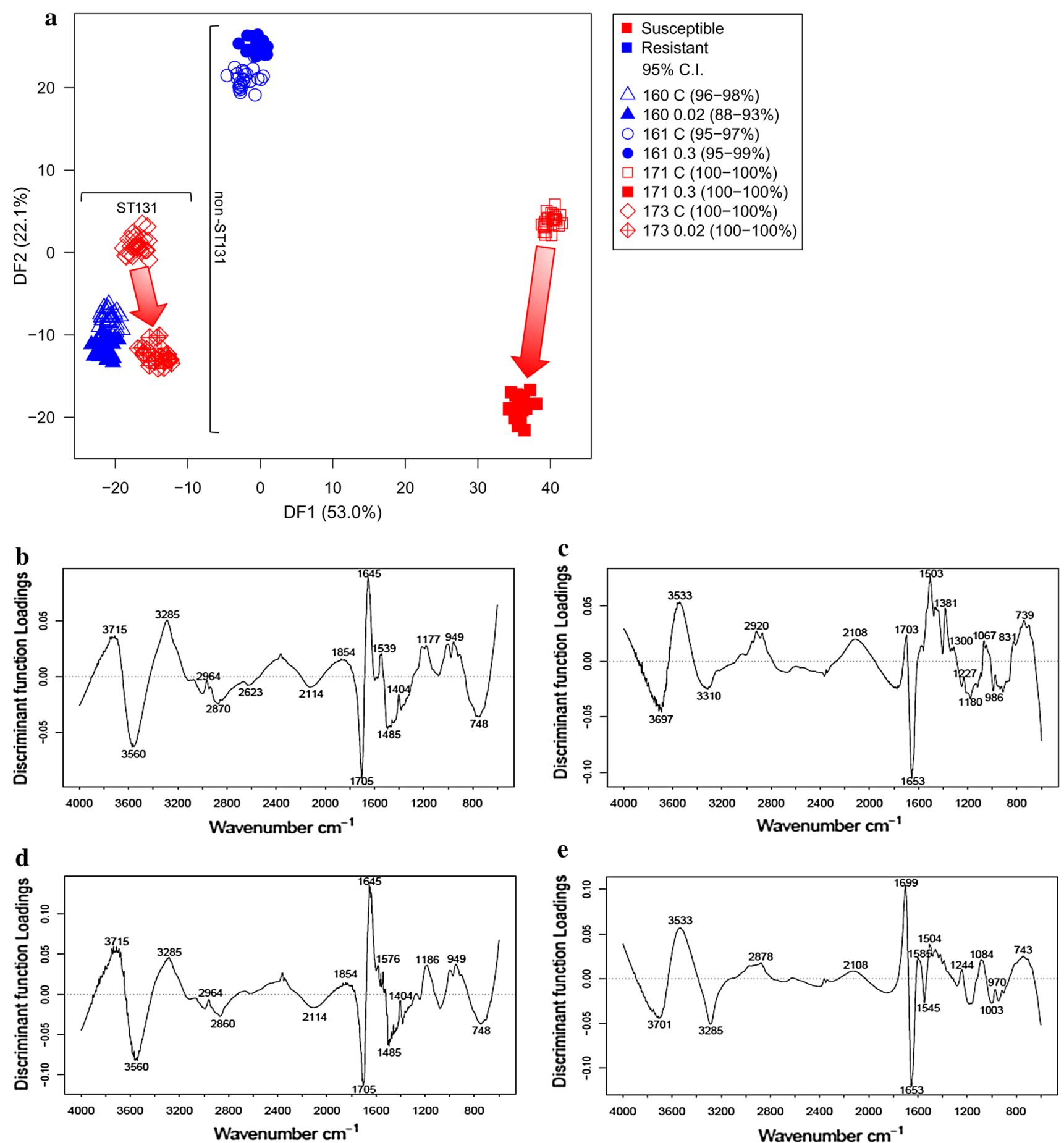

e

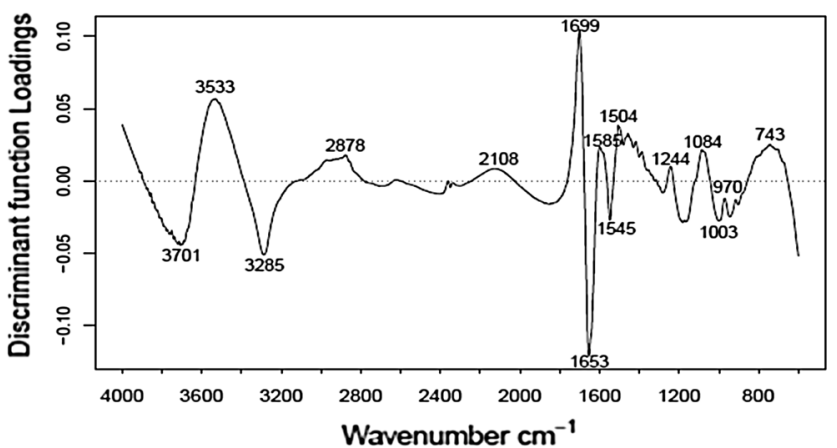

Fig. 3 FT-IR spectroscopy principle component-discriminant function analysis (PC-DFA) of $18 \mathrm{~h}$ Ciprofloxacin challenged and control Escherichia coli. a PC-DFA scores plot (DF1 vs. DF2). PC-DFA loadings plots derived by comparisons of Ciprofloxacin challenged and control samples for each respective isolate b ST131 strain 160

\subsection{Fourier transform infrared (FT-IR) spectroscopy based metabolic fingerprinting}

FT-IR spectroscopy is an established and constantly developing analytical technique, which enables the

(resistant), c ST131 strain 173 (sensitive), d non-ST131 strain 161 (resistant), e non-ST131 strain 171 (intermediate resistance). Within the figures key, $C$ refers to control samples, 0.02 and 0.3 refer to the antibiotic dose $(\mathrm{mg} / \mathrm{l})$, the $95 \%$ confidence Interval $(\mathrm{CI})$ is given in brackets

extremely rapid (seconds per sample), high-throughput (1,000 s of samples per day) and non-destructive analysis of a wide-range of sample types (Ellis and Goodacre 2006; Allwood et al. 2008). FT-IR is also relatively inexpensive and therefore readily lends itself as a rapid first-round 
screening method (Allwood et al. 2008) prior to more expensive and labour intensive approaches. Whilst FT-IR does not provide high levels of functional information, it does enable the collection of a holistic fingerprint that can indicate quantitative changes in peptide, carbohydrate and lipid levels. Therefore, FT-IR could be employed as a first round step where comparisons of non-treated and antibiotic exposed samples are made for numerous bacterial strains and antibiotic combinations. The FT-IR data would then be applied to classify the bacterial strains response as resistant, intermediate resistant, or fully susceptible. An informed selection may then be made to identify which bacterial strains and antibiotic combinations present the most interesting metabolic phenotypes to study in more depth with the lower throughput and financially more expensive chromatography-MS based approaches. The FT-IR data may also be consulted in order to select an appropriate method of chromatography with respect to analysing changes in lipid or polar metabolite composition.

To visualise the distribution of samples on the basis of their IR metabolic fingerprints, a PC-DFA scores plot was generated (Fig. 3a). It was observed that E. coli isolates 160 and 173 (ST131), cluster in close proximity to the negative side of the DF1 axis. E. coli isolate 161 (non ST131) was observed to separate from the ST131 isolates largely along the DF2 axis, which agrees with the results of a previous FT-IR study (AlRabiah et al. 2013, 2014). Interestingly, E. coli isolate 171 (non ST131), which is classed as intermediate resistant, was distantly clustered from all of the other isolates on the positive side of the DF1 axis. The E. coli isolates classed as fully resistant (isolate 160 and 161), revealed very little variance between control and Ciprofloxacin challenged samples, suggesting that on the level of the metabolic fingerprint, very little metabolic change occurs as a result of antibiotic challenge. The sensitive E. coli isolate 173 and the intermediate resistant isolate 171 both showed a clear separation between the control and Ciprofloxacin challenged sample clusters, with the Ciprofloxacin challenged samples clustering to the negative side of the DF2 axis.

As a next step, the PC-DFA loadings were derived on the basis of the wavelengths responsible for the separation of the control samples from the Ciprofloxacin challenged samples for each respective $E$. coli isolate. The fully resistant $E$. coli isolates, 160 and 161, despite being of different sequence type, ST131 and non-ST131 (resulting in their control samples being clearly clustered from each other in the PC-DFA scores plot), revealed very similar spectral changes associated with Ciprofloxacin challenge, suggesting a similar metabolic shift in the response of the two isolates (Fig. 3b, d). The most pronounced spectral changes due to Ciprofloxacin challenge in the fully resistant isolates included peaks at 1,645 and $1,705 \mathrm{~cm}^{-1}$ corresponding to the amide spectral region indicating alterations in protein content in response to Ciprofloxacin challenge. The sensitive $E$. coli isolate 173 and the intermediate resistant isolate 171 also revealed similar spectral changes associated with Ciprofloxacin challenge (Fig. 3c, e). The most pronounced spectral changes due to Ciprofloxacin challenge in these isolates included peaks at 1,653 and $1,700 \mathrm{~cm}^{-1}$ again corresponding to the amide spectral region. Although the preliminary sample screening approach revealed significant protein alterations, this is perhaps unsurprising given that Ciprofloxacin and other fluoroquinolones are predicted to target enzymatic proteins such as DNA topoisomerase II and IV, therefore it may be expected that alterations in protein content would dominate the alterations observed in the lipid, polysaccharide and mixed spectral regions of the IR spectra. Given the tight clustering of samples of the same experimental class within the PC-DFA scores plot (Fig. 3a), it was clear that the within experiment technical reproducibility of sample growth and collection was high and therefore likely suited to UHPLC-MS lipid profiling.

\subsection{Reverse phase ultra high performance liquid chromatography-mass spectrometry (UHPLC-MS) based lipid profiling}

Following UHPLC-MS analysis in both ESI positive and negative ionisation modes, peak deconvolution was performed using the freely available XCMS software (http:// masspec.scripps.edu/xcms/xcms.php) (Dunn et al. 2008; Wedge et al. 2011), producing a MS Excel based XY matrix of mass spectral features (with related accurate $\mathrm{m} / \mathrm{z}$ and retention time variable pairs) $\times$ sample, with peak area inputted where the mass spectral feature was detected in each sample. In the UHPLC-MS ESI positive mode and negative mode datasets a total of 2,933 and 1,505 mass spectral features were deconvolved respectively, features showing greater than $20 \%$ relative standard deviation within quality control (QC) samples were removed as were features detected in the first $0.5 \mathrm{~min}$ of LC (void volume), resulting in a total of 2,642 and 1,317 features in ESI positive and negative modes respectively.

Following deconvolution, the putative identification of lipid features was performed applying the PUTMEDIDLCMS set of workflows (Brown et al., 2011) (Table S2). An initial appraisal of the putatively identified lipid species suggested that the phospholipids of E. coli are predominantly phosphatidyl cholines (PCs) and/or phosphatidyl ethanolamines (PEs). Given previous reports that state the major lipid classes of $E$. coli are represented by PEs, phosphatidyl glycerols (PGs) and Cardiolipins, with minor contributions made by phosphatidyl serines (PSs) and phosphatidic acids (PAs) (Ames 1968), it is perhaps tempting to speculate that the major lipid species detected in this study are more likely to be PEs than PCs. The PC 

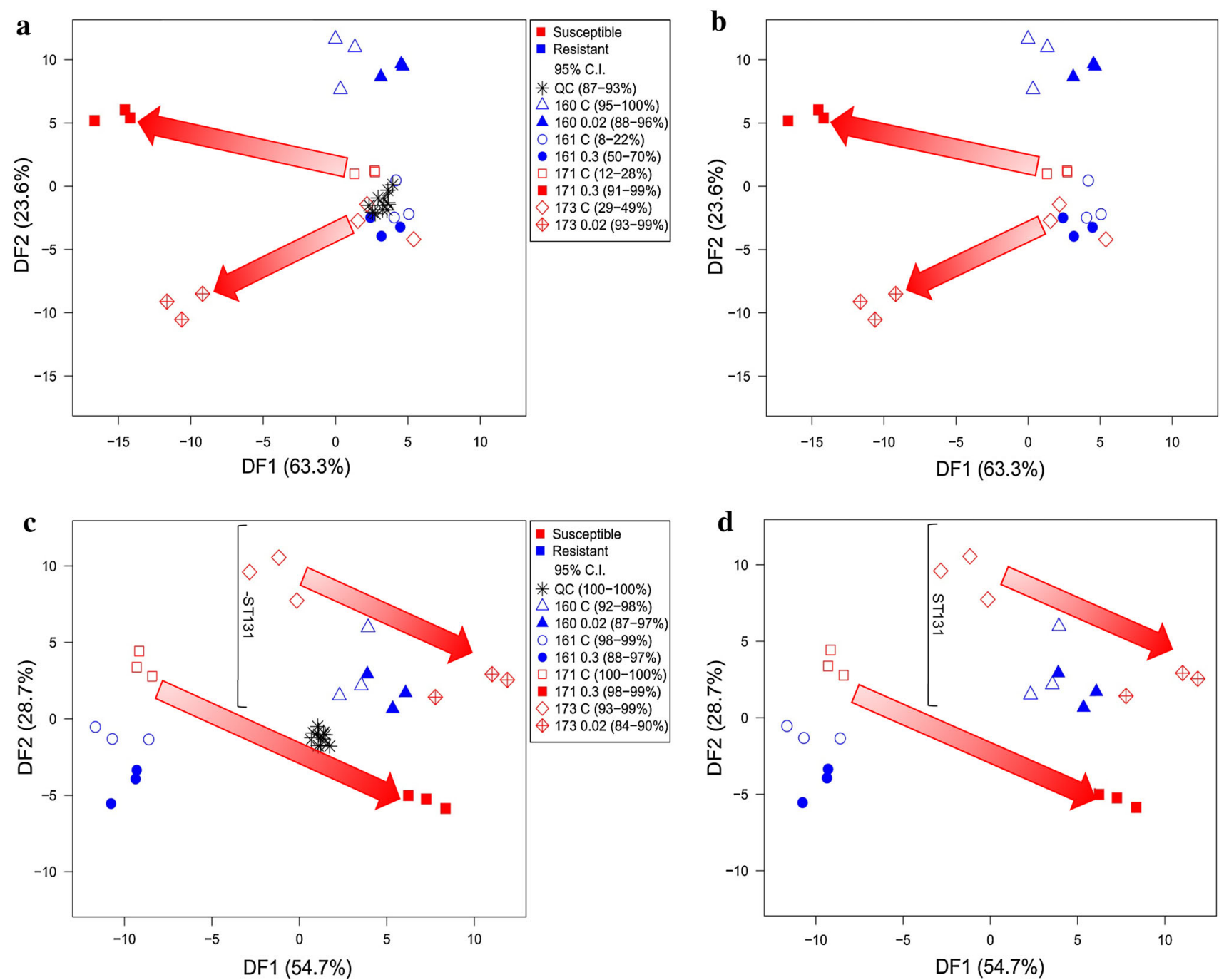

Fig. 4 UHPLC-MS principle component-discriminant function analysis (PC-DFA) of $18 \mathrm{~h}$ Ciprofloxacin challenged and control Escherichia coli. PC-DFA scores plot (DF1 vs. DF2) of UHPLCMS ESI + mode data a with QCs included and $\mathbf{b}$ with QCs removed,

and/or PE phospholipids were largely detected in ESI positive ionisation mode although a smaller number were also observed in ESI negative mode. Further, a small number of PAs were observed in ESI positive and negative modes and a number of PGs were observed predominantly in ESI negative mode. Further to phospholipids, a large number of acylglycerols and free fatty acids were detected, as well as further diverse lipid species such as sterols, ceramides and sphingolipids. The putative level of identification provided by this approach can be categorised as identification levels 2 and 3 according to the Metabolomics Standards Initiative guidelines (Sumner et al. 2007).

As a first step towards the appraisal of the UHPLC-MS datasets, PC-DFA was applied and score plots were generated (Fig. 4) in order to visualise the distribution of the various samples and sample groups in relation to each other.

PC-DFA scores plot (DF1 vs. DF2) of UHPLC-MS ESI- mode data c with QCs included and d with QCs removed. Within the figures key, $C$ refers to control samples, 0.02 and 0.3 refer to the antibiotic dose $(\mathrm{mg} / \mathrm{l})$, the $95 \%$ confidence interval (CI) is given in brackets

The central positioning of the QC samples through the 0 axes of both DF1 and DF2 (Fig. 4a, c), indicate that after deconvolution and quality control steps, the QC samples still represent an average data point, and that the levels of analytical and technical reproducibility were high. Similar to observations within the FT-IR dataset when subjected to PC-DFA (Fig. 3), the UHPLC-MS datasets also indicated that alterations due to Ciprofloxacin challenge within the resistant bacterial isolates (isolates 160 and 161), resulted in only a small level of sample separation within the PC-DFA score plots for both ESI positive (Fig. 4a, b) and negative (Fig. 4c, d) ionisation modes. Perhaps of the greatest significance was the clear differentiation between control and Ciprofloxacin challenged samples of the sensitive E. coli isolate 173 and the intermediate resistant $E$. coli isolate 171 , with lipid changes due to Ciprofloxacin challenge being associated 
a
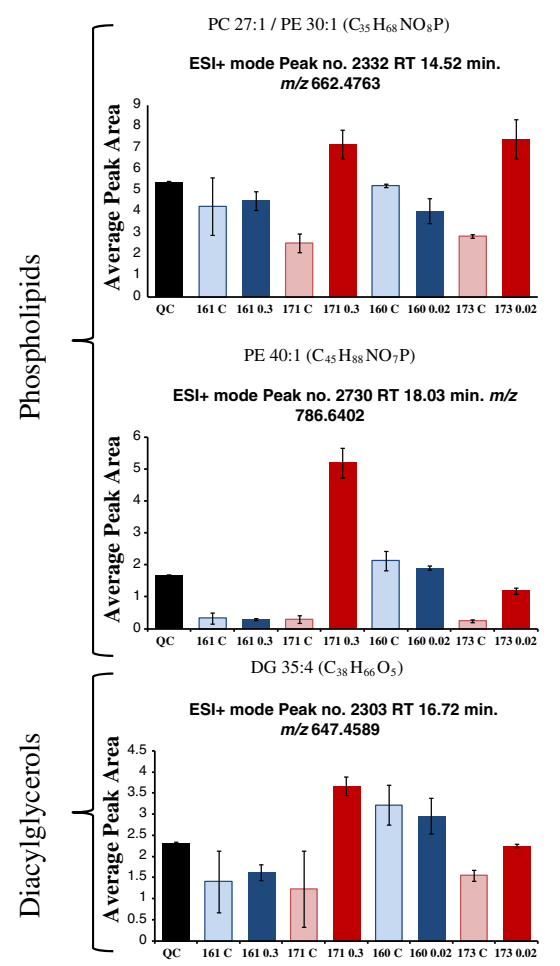

b

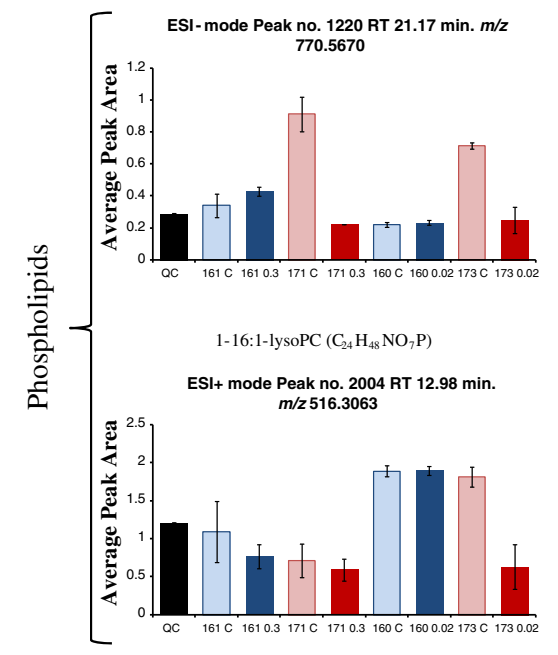

c

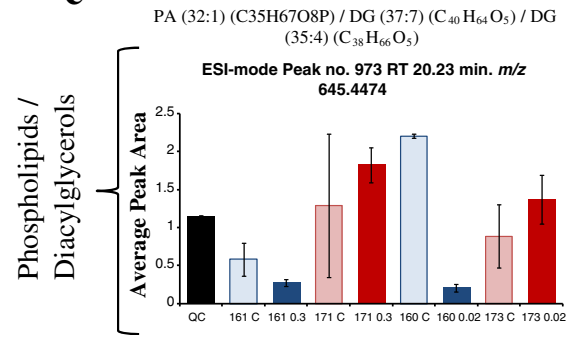

PC 29:1 / PE 32:1 $\left(\mathrm{C}_{37} \mathrm{H}_{72} \mathrm{NO}_{8} \mathrm{P}\right) /$ PA 34:2

$\left(\mathrm{C}_{37} \mathrm{H}_{69} \mathrm{O}_{8} \mathrm{P}\right) /$
PE-NMe $(\mathrm{O}-28: 0)\left(\mathrm{C}_{34} \mathrm{H}_{72} \mathrm{NO}_{6} \mathrm{P}\right)$

ESI+ mode Peak no. 2398 RT $14.97 \mathrm{~min}$.

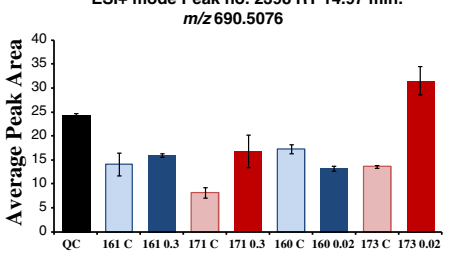

PC (29:1) / PE (32:1) $\left(\mathrm{C}_{37} \mathrm{H}_{72} \mathrm{NO}_{8} \mathrm{P}\right)$

ESI-mode Peak no. 1050 RT $19.71 \mathrm{~min} . \mathrm{m} / \mathrm{z}$

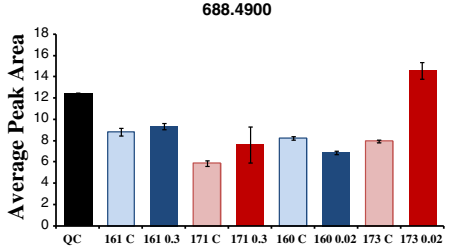

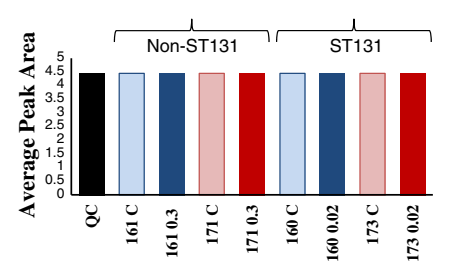

PE 34:1/ PC 31:1 ( $\left.\mathrm{C}_{30} \mathrm{H}_{76} \mathrm{NO}_{8} \mathrm{P}\right) / \mathrm{PA} 36: 2$

$\left(\mathrm{C}_{39} \mathrm{H}_{73} \mathrm{O}_{8} \mathrm{P}\right) / \mathrm{PC}(\mathrm{O}-28: 0)\left(\mathrm{C}_{36} \mathrm{H}_{76} \mathrm{NO}_{6} \mathrm{P}\right)$

ESI+ mode Peak no. 2479 RT $15.46 \mathrm{~min} . \mathrm{m} / \mathrm{z}$

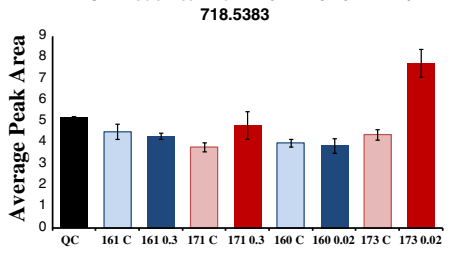

PE-NMe $(\mathrm{O}-28: 0)\left(\mathrm{C}_{34} \mathrm{H}_{72} \mathrm{NO}_{6} \mathrm{P}\right)$ :

ESI+ mode Peak no. 2458 RT 12.67 min.

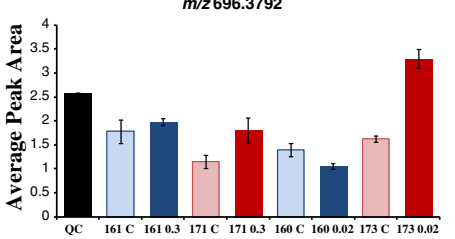

$\mathrm{PA}(38: 4)$ (C41H73O8P) / PS (32:3) $\left(\mathrm{C}_{38} \mathrm{H}_{68} \mathrm{NO}_{10} \mathrm{P}\right) /$ 2-octadecanoyl -1-hexadecyl-sn-glycero-3-phosphate $\left(\mathrm{C}_{37} \mathrm{H}_{75} \mathrm{O}_{7} \mathrm{P}\right)$

PC 32:2 / PC O-32:3 $\left(\mathrm{C}_{40} \mathrm{H}_{76} \mathrm{NO}_{7} \mathrm{P}\right) /$

1-(6-[5]-ladderane-hexanyl)-2-(8-[3]--ladderane-octanyl)-snglycerophosphoethanolamine $\left(\mathrm{C}_{43} \mathrm{H}_{72} \mathrm{NO}_{6} \mathrm{P}\right)$

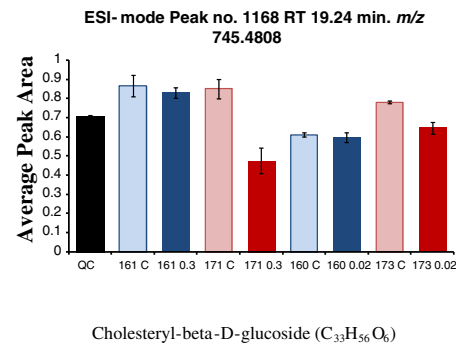

ESI+ mode Peak no. 2737 RT 15.25 min. m/z 788.4753

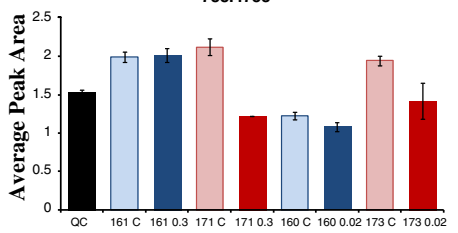

17-Hydroxy-3-oxo-17alpha-pregna-1,4-diene-21-carboxylic acid, gamma-lactone / Canrenone / Norethindrone acetate / Norethisterone

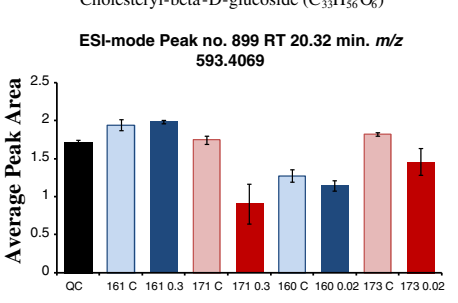
acetate $\left(\mathrm{C}_{22} \mathrm{H}_{28} \mathrm{O}_{3}\right)$ : Steroid Derivatives
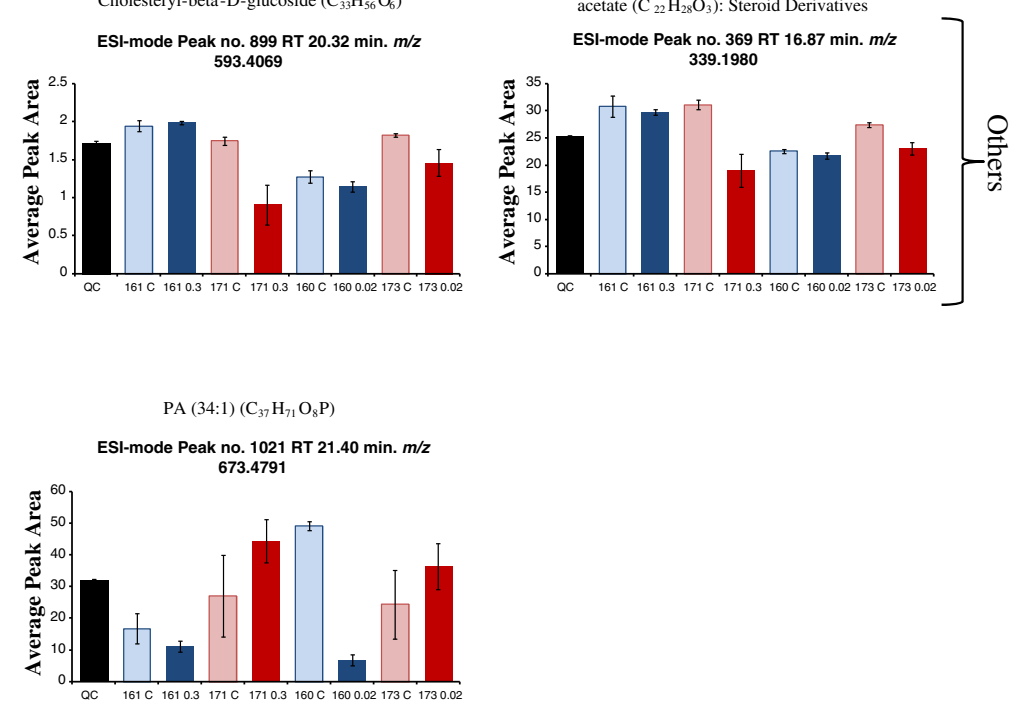
4Fig. 5 UHPLC-MS trend plots of lipids significantly altered in response to Ciprofloxacin: Lipids differentially regulated between susceptible and resistant isolates. a Significantly up-regulated in susceptible isolates. b Significantly down-regulated in susceptible isolates. c Significantly down-regulated in resistant isolates. Error bars represent the standard error within the non averaged data for each experimental class

with a trajectory across the DF1 axis for both ESI positive (Fig. 4a, b) and negative (Fig. 4c, d) ionisation modes. The PC-DFA scores plots of the UHPLC-MS datasets overall indicate that whilst there are alterations in lipid profiles of the resistant bacterial isolates (isolates 160 and 161) when challenged by Ciprofloxacin, these are overall minimal in nature compared to the vast alterations in lipid profiles resulting from Ciprofloxacin challenge within the sensitive and intermediate resistant isolates (isolates 173 and 171 respectively).

Following PC-DFA, the next objective was to select which lipids were significantly altered between control and Ciprofloxacin challenged samples for each bacterial isolate independently, by applying significance testing. Two significance tests were applied and a consensus drawn between them. Firstly, a PLS algorithm was applied to the data and the mass spectral features were sorted by the magnitude of their corresponding PLS coefficient values. Secondly, the ANOVA statistical test was applied to all mass spectral features. Finally, starting with the mass spectral feature with the highest PLS coefficient, the top 50 were selected whose p-value computed by ANOVA was less than 0.05 (Table S3 and S4). For each bacterial isolate, the significant features were sorted according to retention time, since certain lipid species were represented by multiple mass spectral features, the top 50 list was next reduced to just the major ion (most intense) observed for each putatively identified lipid species. The top 50 mass spectral features lists for each isolate and ESI polarity, after having been reduced in this manner, were next compiled and a total list of significant lipid mass spectral features across all bacterial isolates was obtained. As a next step, trend plots were generated for each of the significant lipid features, which were then grouped according to their response to Ciprofloxacin: lipid features that were commonly up- or down-regulated across both resistant and sensitive $E$. coli isolates (Fig. S1); lipid features that were up- or down-regulated specifically in susceptible or in resistant isolates (Fig. 5); lipid features that were differentially regulated between resistant and sensitive isolates (Fig. 6).

3.4 A wide selection of $E$. coli lipid species are altered by challenge with Ciprofloxacin, with many showing differential regulation between sensitive and resistant $E$. coli isolates

After the statistically significant lipids had been grouped according to their response under Ciprofloxacin challenge, the patterns of response were considered taking into account the putative assignment of the lipid species and the levels of saturation within the fatty acid components of the lipid species, in order to identify potentially significant trends within the results. Figure S1 reveals a series of lipid alterations that were grouped as showing common responses between both resistant and sensitive isolates. Typically, higher levels of lipid up-regulation were observed within the sensitive isolate 173 and intermediate resistant isolate 171 , with the lipids being putatively assigned as PAs, PGs, PAs or PGs, and PGs or DGs (Diacyglycerides). Again, for the lipid species that were downregulated, typically higher levels of down-regulation were observed in the sensitive isolate 173 and intermediate resistant isolate 171 , with the lipids being putatively assigned as PC or PE or PE-NmE (n-Methyl PE) and one lipid putatively assigned as a highly unsaturated DG with total chain composition of 40:10 or 38:7 (dependent on the potential ESI adduct that is formed). The phospholipids that were generally up-regulated, according to their putative assignments, had more saturated fatty acids (typically one or two unsaturated bonds) than the phospholipids that were down regulated which typically had two-to-four unsaturated bonds within the fatty acids.

The lipid features that were up- or down-regulated specifically in susceptible or in resistant isolates are presented in Fig. 5. The lipids that were significantly up-regulated in the sensitive isolates generally showed a higher level of up-regulation within the fully sensitive isolate 173 than the intermediate resistant isolate 171. A total of five phospholipids assigned as PEs or PCs were up-regulated, the putative assignments suggest high levels of saturation within the phospholipid fatty acids, with only one or two unsaturated bonds being present. The lipids that were significantly down-regulated specifically in the sensitive isolates generally showed similar levels of down-regulation between the fully sensitive isolate 173 and the intermediate resistant isolate 171. A small number of phospholipids fell within this response grouping and were putatively assigned as PCs or PEs and PAs or PSs. A lyso-PC and Cholesterylbeta-D-glucoside were also observed to be down-regulated. Only a small number of lipids were altered specifically within the resistant isolates 160 and 161, all of which were highly down-regulated upon challenge with Ciprofloxacin, these ions were putatively assigned as DGs and PAs.

Perhaps the most interesting series of lipid alterations observed within the investigation, were those that were differentially regulated between the sensitive and resistant isolates (Fig. 6). A large number of phospholipids were found to be down-regulated in the resistant isolates (isolates 160 and 161) and up-regulated in the sensitive and intermediate resistant isolates 173 and 171. Most commonly the significant lipids were down-regulated in the 
$\mathbf{a}$

PC (32:0) / PE (35:0) / PE-NMe(34:0) (C $\left.{ }_{40} \mathrm{H}_{80} \mathrm{NO}_{8} \mathrm{P}\right) /$

$\mathrm{N}$-(2-hydroxytricosanoyl)-4,8-sphingadienine
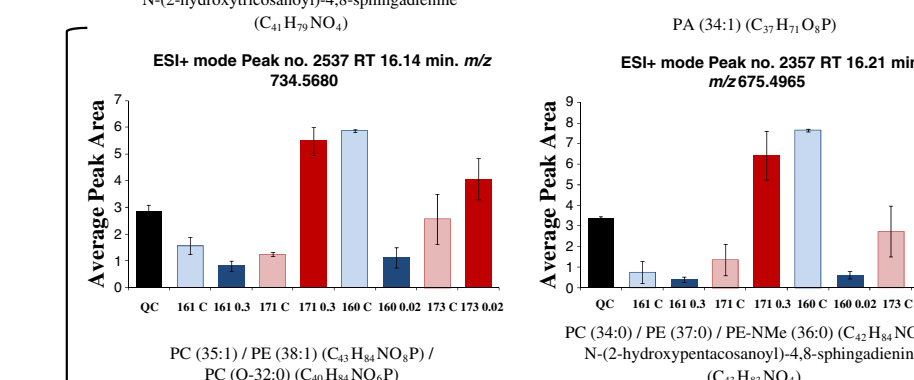
$\mathrm{PC}(14: 0 / \mathrm{dm} 18: 0) / \mathrm{PC}(16: 0 / \mathrm{dm} 16: 0) /$
$\mathrm{PC}(\mathrm{O}-32: 1)\left(\mathrm{C}_{40} \mathrm{H}_{80} \mathrm{NO}_{7} \mathrm{P}\right)$
ESI+ mode Peak no. $2480 \mathrm{RT} 16.50 \mathrm{~min} . \mathrm{m} / \mathrm{z}$

El+ mode Peak no. 2357 RT $16.21 \mathrm{~min}$.

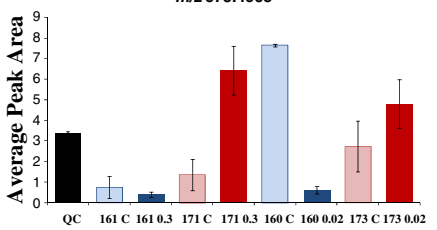

$\mathrm{PC}(34: 0) / \mathrm{PE}(37: 0) / \mathrm{PE}-\mathrm{NMe}(36: 0)\left(\mathrm{C}_{42} \mathrm{H}_{84} \mathrm{NO}_{8} \mathrm{P}\right) /$

$\mathrm{N}$-(2-hydroxypentacosanoyl)-4,8-sphingadienine

$\left(\mathrm{C}_{43} \mathrm{H}_{83} \mathrm{NO}_{4}\right)$

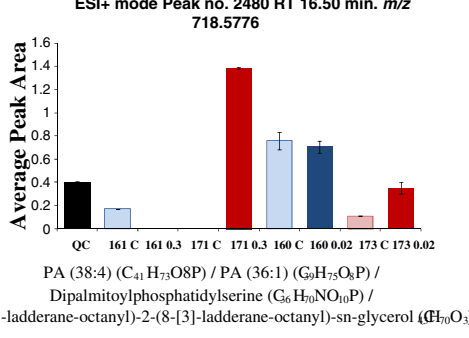

ESI+ mode Peak no. 2688 RT $17.45 \mathrm{~min} . \mathrm{m} / \mathrm{z}$ 774.6015
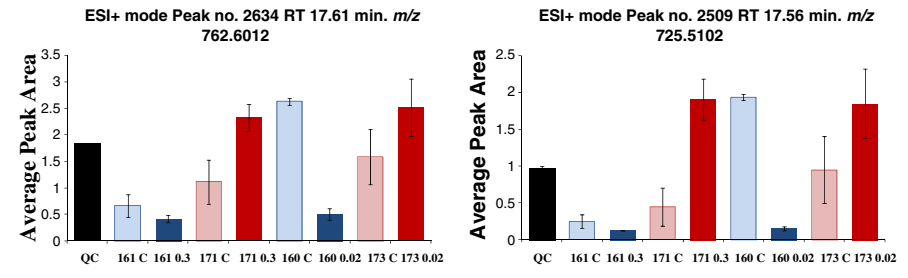

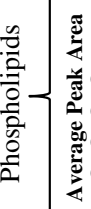
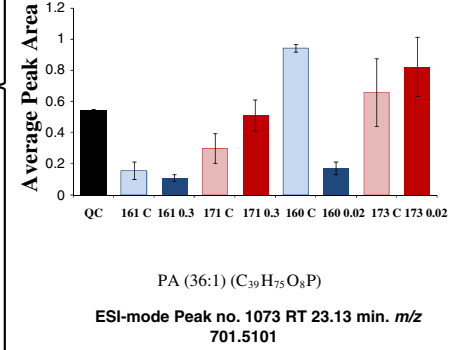

PA (36:2) $\left(\mathrm{C}_{39} \mathrm{H}_{73} \mathrm{O}_{8} \mathrm{P}\right) / \mathrm{DG}(39: 5)\left(\mathrm{C}_{2} \mathrm{H}_{72} \mathrm{O}_{5}\right) /$ 1-(10-methyl-

hexadecanoyl-2-(8-[3]-ladderane-octanyl)-sn-glycerol / 1-(9,14-dimethyl-

pentadecanoyl-2-(8-[3]-ladderane-octanyl)-sn-glycerol $\left(6 \mathrm{H}_{72} \mathrm{O}_{4}\right)$

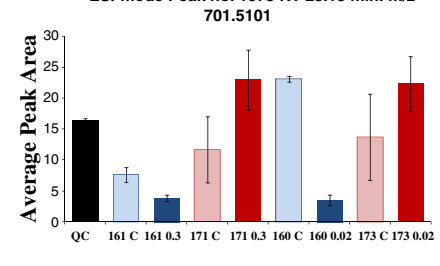

ESI-mode Peak no. 1068 RT $21.69 \mathrm{~min} . \mathrm{m} / \mathrm{z}$
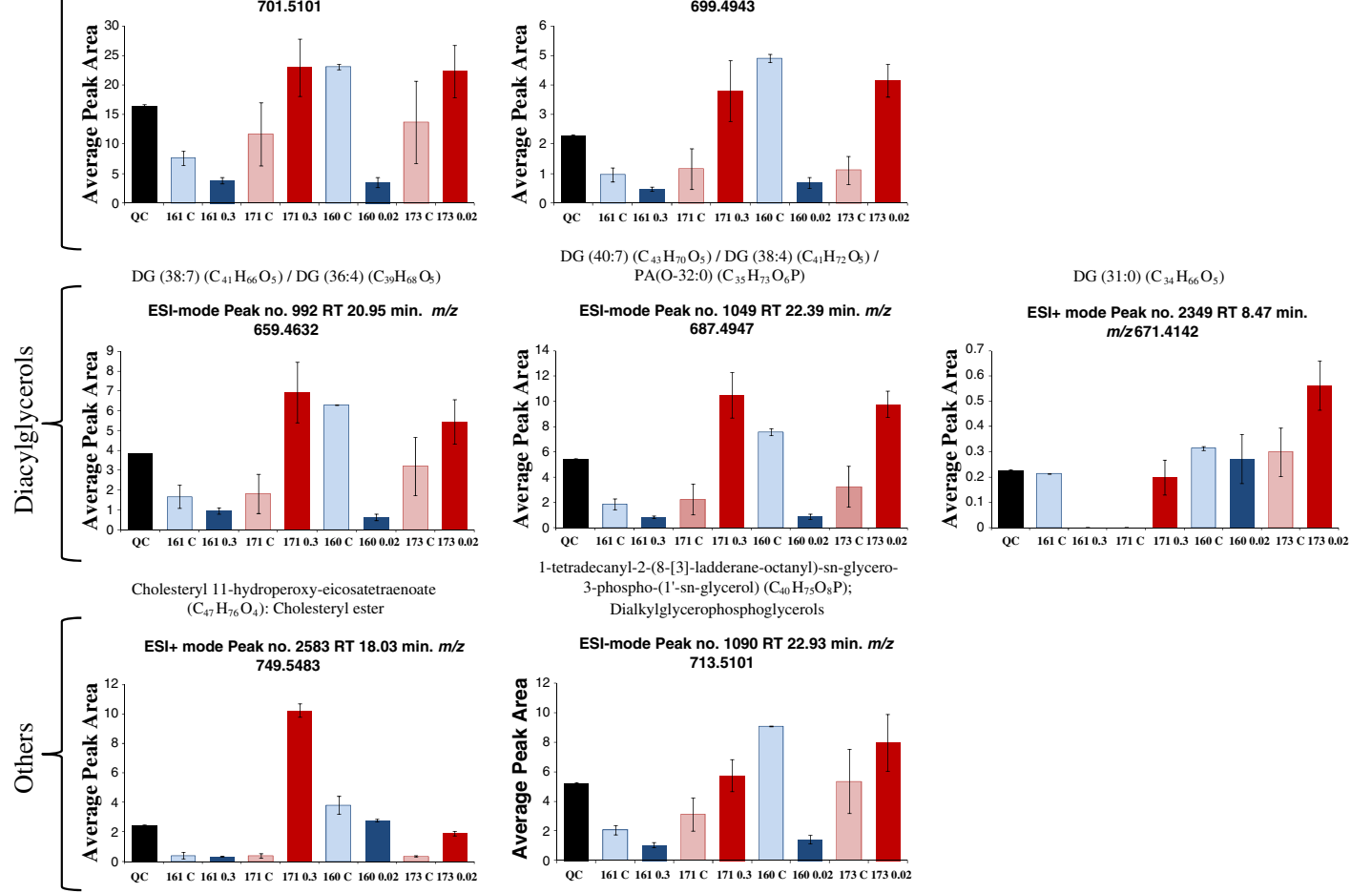

b

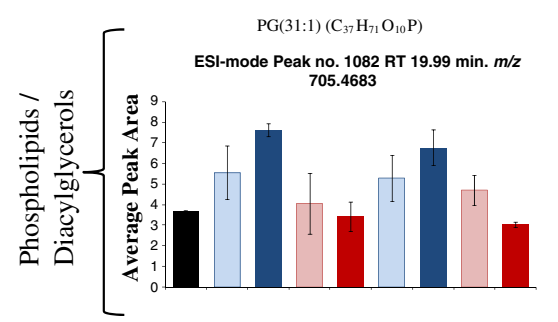
PG (30:0) $\left(\mathrm{C}_{36} \mathrm{H}_{71} \mathrm{O}_{10} \mathrm{P}\right) / \mathrm{PA}(32: 0)\left(\mathrm{C}_{35} \mathrm{H}_{69} \mathrm{O}_{8} \mathrm{P}\right) / \mathrm{DG}$
$(37: 6)\left(\mathrm{C}_{40} \mathrm{H}_{66} \mathrm{O}_{5}\right) ; \mathrm{DG}(35: 3)\left(\mathrm{C}_{38} \mathrm{H}_{68} \mathrm{O}_{5}\right)$ ESI-mode Peak no. 1059 RT $20.05 \mathrm{~min}$. $\mathrm{m} / \mathrm{z}$
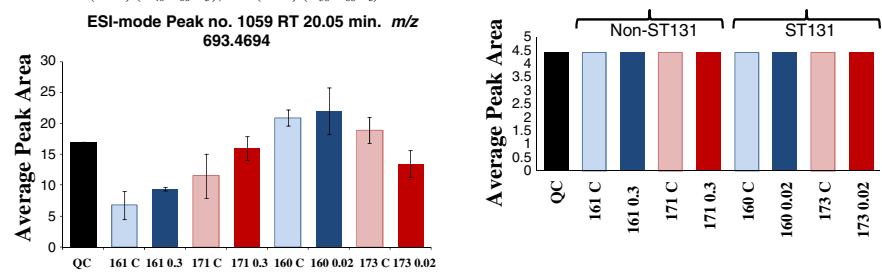
4Fig. 6 UHPLC-MS trend plots of lipids significantly altered in response to Ciprofloxacin: Lipids specifically regulated in susceptible or resistant isolates. a Up-regulated in susceptible isolates and down regulated in resistant isolates. b Up-regulated in resistant isolates and down regulated in susceptible isolates. Error bars represent the standard error within the non averaged data for each experimental class

resistant isolates and up-regulated in the sensitive isolate, with the majority of the significant lipids being putatively assigned as PCs or PEs and PAs. A small number of DGs also fell within this response class, with putative assignments suggesting potentially high levels of un-saturation within the fatty acid components. Interestingly, an ion putatively assigned as Cholesteryl 11-hydroxy-eicosatetraenoate was extremely highly up-regulated, but only within the intermediate resistant isolate 171, perhaps indicating an alternative mode of antibiotic exclusion to that observed within the resistant isolates 160 and 161. In comparison, only a very small number of lipids were observed to be up-regulated within the resistant isolates and down-regulated within the sensitive isolate. One such ion was putatively assigned as a PG with highly saturated fatty acids and the other as being either a PA or PG again with highly saturated fatty acids.

Several trends have emerged within the LC-MS lipidomics dataset with the most significant perhaps being that all phospholipids were assigned as PCs or PEs and PAs or PGs in class, with their putative assignments suggesting high levels of saturation within the fatty acids. Alternatively, a large number of ions putatively assigned as DGs were shown to be significant, many of the putatively assigned DGs possessed more unsaturated fatty acids than those observed for the phospholipids. Typically the levels of up- or down-regulation observed for the sensitive isolate 173 and intermediate resistant isolate 171 were much higher than the levels of regulation observed within the resistant isolates, as indicated also by PC-DFA (Fig. 4). Perhaps of further interest, is that the majority of statistically significant lipids, were up-regulated in the sensitive isolate whilst being down-regulated in the resistant isolates, with only small numbers of significant lipids revealing the converse trend. It appears that the resistant isolates are largely characterised by down-regulation of PCs or PEs in response to Ciprofloxacin challenge, whereas the sensitive isolates show high levels of up-regulation of PCs or PEs in response to Ciprofloxacin challenge, perhaps suggesting that high levels of lipid membrane modulation are taking place as the antibiotic enters and takes effect upon the bacterial cell. The developed experimental design has accounted that the only difference between control and Ciprofloxacin challenged samples for each respective strain is the presence of the antibiotic. Therefore it is tempting to make the assumption that all lipid changes are in direct response to the effects exerted on the bacterial cell by the antibiotic. However, previous research has shown that fatty acid alterations can occur as a response to environmental changes including temperature, $\mathrm{pH}$, and osmotic pressure, a phenomena known as phenotypic shift (Crompton et al. 2014). Therefore, with respect to the current study, it must be considered that whilst some of the observed changes in lipid composition in response to Ciprofloxacin may be the result of the antibiotics interaction with the phospholipid membrane, or in the case of resistant strains, the antibiotics exclusion via membrane transport, other lipid alterations may be indirect responses due to the antibiotic altering environmental conditions such as $\mathrm{pH}$ within the bacterial culture resulting in phenotypic shift.

Given that previous research has suggested that the major phospholipid species detected in E. coli are PEs (Arnes 1968), it is perhaps unsurprising that the majority of lipids that were significantly altered by Ciprofloxacin challenge within this study were putatively assigned as being PEs or PCs. This may suggest that Ciprofloxacin treatment is specifically affecting PEs or PCs, or alternatively that these are the major lipid species of $E$. coli and thus are more likely to have changes in their levels detected due to their higher concentrations and that the alternate species of phospholipids may be close to or under the detection limit of the employed LC-MS instrumentation. However, if the latter was the case, then perhaps one would expect to also observe alterations in the other major lipid classes of $E$. coli, namely PGs and Cardiolipins (Arnes 1968). Several previous investigations, although based upon extracted liposomes (Bensikaddour et al. 2008) or extracted membrane lipids (Leying et al. 1986; Merino et al. 2003) of E. coli, rather than on the bacterial cells within culture as performed in this study, also suggested that Ciprofloxacin interacts at the water-phospholipid head group interface of the lipid membrane as revealed by atomic force microscopy (Merino et al. 2003) and also by combination of quasi-elastic light scattering and steady-state fluorescence anisotropy, combined with attenuated total reflectance (ATR)-FT-IR and ${ }^{31} \mathrm{P}$ NMR spectroscopy (Bensikaddour et al. 2008), with the studies suggesting that re-modulation of the phospholipid fatty acids takes place rather than a conversion of headgroup thus forming a different class of phospholipid. Taking these observations into account, it is clearly of much importance that for the approach applied in this study utilising the high sensitivity of LC-LTQ Orbitrap MS to investigate lipid alterations within the "true" bacterial cell to be of greater value, it will be of massive significance to develop online LC-MS/MS methods and offline MS $^{n}$ (van der Hooft et al. 2011, 2012; Rojas-Cherto et al. 2012; Roux et al. 2012) analyses of pre-fractionated samples, in order to not just unambiguously assign a lipid identification, but also to identify the levels of saturation and positions of unsaturated 
bonds within fatty acids. Once developed, such methods and their unrivalled abilities to unambiguously confirm the identifications of the lipids and elucidate unsaturated fatty acid bond positions will provide the highest levels of information currently possible and will aid greatly our understanding of the effects of Ciprofloxacin modulation of fatty acid structure within the putatively assigned PE and PC phospholipids.

\section{Concluding remarks}

The LC-MS workflow developed for the analysis of lipid species within $E$. coli when under the challenge of an antibiotic such as Ciprofloxacin, has been shown to be a technically and analytically reproducible method that is useful for discovery phase studies on bacterial modes of antibiotic resistance. Further, by combining a lipid profiling approach with alternative metabolomics platforms suited to profiling primary metabolism (e.g. GC-MS: Winder et al. 2008), the approach may be highly successful for studying the mechanisms underlying antibiotic modes of action within sensitive isolates, as well as mechanisms of resistance. Further, within this study, the developed workflow has been shown to identify a range of interesting and potentially clinically significant lipid alterations in E. coli upon challenge with Ciprofloxacin. However, to obtain a more satisfactory level of information, the next step is the development of online RP-UHPLC-MS/MS and offline direct infusion $\mathrm{MS}^{n}$ methods with the LTQ-Orbitrap XL MS system for identification of lipid species across all classes. Such an approach will allow identification of phospholipid classes and fatty acid constituents by employing orthogonal Retention Time information and MS/MS spectral fragmentation compared to analytical standards. By further employing online fraction collection and performing $\mathrm{MS}^{3>5}$ level experiments employing collision induced dissociation and/or higher energy collision dissociation methods then the positions of the unsaturated bond positions within the fatty acid constituents of phospholipids may potentially be identified. The current study has suggested the great potential of LC-MS lipidomics for the study of antibiotic modes of action or bacterial modes of resistance, although greater abilities to unambiguously identify the lipid species are required to maximise the biological information content obtained by such a nontargeted lipidomics approach.

Acknowledgments JWA, AV, YX, and RG would like to acknowledge CR-UK for current research funding. HR thanks The Saudi Ministry of higher education and King Saud University for funding. EC and RG are grateful to the EU Commonsense (http:// www.fp7projectcommonsense.eu/) project (Grant 261809) financed by the European Commission under the 7th Framework Programme for Research and Technological Development.

\section{References}

Allwood, J. W., Clarke, A., Goodacre, R., \& Mur, L. A. J. (2010) Dual metabolomics: A novel approach to understanding plantpathogen interactions. Phytochem., 71, 590-597.

Allwood, J. W., Ellis, D. I., \& Goodacre, R. (2008). Metabolomic technologies and their application to the study of plants and plant-host interactions. Physiologia Plantarum, 132, 117-135.

Allwood, J. W., Ellis, D. I., Heald, J. K., Goodacre, R., \& Mur, L. A. J. (2006). Metabolomic approaches reveal that phosphatidic and phosphatidyl glycerol phospholipids are major discriminatory metabolites in responses by Brachypodium distachyon to challenge by Magnaporthe grisea. Plant Journal, 46, 351-368.

Allwood, J. W., Erban, A., de Koning, S., Dunn, W. B., Luedemann, A., Lommen, A., et al. (2009). Inter-laboratory reproducibility of fast gas chromatography-electron impact-time of flight mass spectrometry (GC-EI-TOF/MS) based plant metabolomics. Metabolomics, 5, 479-496.

AlRabiah, H., Correa, E., Upton, M., \& Goodacre, R. (2013). Highthroughput phenotyping of uropathogenic $E$. coli isolates with Fourier transform infrared spectroscopy. The Analyst, 138, 1363-1369.

AlRabiah, H., Xu, Y., Rattray, N. J. W., Vaughan, A. A., Gibreel, T., Sayqal, A., et al. (2014). Multiple metabolomics of uropathogenic E. coli reveal different information content in terms of metabolic potential compared to virulence factors. The Analyst. doi:10.1039/c4an00176a.

Ames, G. F. (1968). Lipids of Salmonella typhimurium and Escherichia coli: Structure and metabolism. Journal of Bacteriology, 95, 833-843.

Bensikaddour, H., Snoussi, K., Lins, L., Van Bambeke, F., Tulkens, P. M., Brasseur, R., et al. (2008). Interactions of ciprofloxacin with DPPC and DPPG: Fluorescence anisotropy, ATR-FTIR and ${ }^{31} \mathrm{P}$ NMR spectroscopies and conformational analysis. Biochimica et Biophysica Acta, 1778, 2535-2543.

Biais, B., Allwood, J. W., Deborde, C., Xu, Y., Maucort, M., Beauvoit, B., et al. (2009). ${ }^{1} \mathrm{H}-\mathrm{NMR}$, GC-EI-TOF/MS, and dataset correlation for fruit metabolomics: application to spatial metabolite analysis in melon. Analytical Chemistry, 81, 2884-2894.

Bligh, E. G., \& Dyer, W. J. (1959). A rapid method of total lipid extraction and purification. Canadian Journal of Biochemistry and Physiology, 37, 811-917.

Brown, M., Wedge, D., Goodacre, R., Kell, D. B., Baker, P. N., Kenny, L. C., et al. (2011). Automated workflows for accurate mass-based putative metabolite identification in LC/MS-derived metabolomic datasets. Bioinformatics, 27, 1108-1112.

Castrillo, J. I., Zeef, L. A., Hoyle, D. C., Zhang, N., Hayes, A., Gardner, D. C. J., et al. (2007). Growth control of the eukaryote cell: A systems biology study in yeast. Journal of Biology, 6, 4.

Crompton, M. J., Dunstan, R. H., Macdonald, M. M., Gottfries, J., von Eiff, C., \& Roberts, T. K. (2014). Small changes in environmental paramaters lead to alterations in antiobiotic resistance, cell morphology, and membrane fatty acid composition in Staphylococcus lugdunensis. PLoS ONE, 9(4), e92296.

De Vos, C. H. R., Moco, S., Lommen, A., Keurentjes, J. J. B., Bino, R. J., \& Hall, R. D. (2007). Untargeted large-scale plant metabolomics using liquid chromatography coupled to mass spectrometry. Nature Protocols, 2, 778-791. 
Diederen, B. M., \& Kluytmans, J. A. (2006). The emergence of infections with community-associated methicillin resistant Staphylococcus aureus. The Journal of Infection, 52, 157-168.

Dunn, W. B., Broadhurst, D., Begley, P., Zelena, E., FrancisMcIntyre, S., Anderson, N., et al. (2011). Procedures for largescale metabolic profiling of serum and plasma using gas chromatography and liquid chromatography coupled to mass spectrometry. Nature Protocols, 6, 1060-1083.

Dunn, W. B., Broadhurst, D., Brown, M., Baker, P. N., Redman, C. W. G., Kenny, L. C., et al. (2008). Metabolic profiling of serum using ultra performance liquid chromatography and the LTQorbitrap mass spectrometry system. Journal of Chromatography B, 871(2), 288-298.

Dunn, W. B., Broadhurst, D. I., Deepak, S. M., Buch, M. H., McDowell, G., Spasic, I., et al. (2007). Serum metabolomics reveals many novel metabolic markers of heart failure, including pseudouridine and 2-oxoglutarate. Metabolomics, 3, 413-426.

Ellis, D. I., \& Goodacre, R. (2006). Metabolic fingerprinting in disease diagnosis: biomedical applications of infrared and Raman spectroscopy. Analyst, 131, 875-885.

Fiehn, O. (2002). Metabolomics-The link between genotypes and phenotypes. Plant Molecular Biology, 48, 155-171.

Fiehn, O., Kopka, J., Dörmann, P., Altmann, T., Trethewey, R. N., \& Willmitzer, L. (2000). Metabolite profiling for plant functional genomics. Nature Biotechnology, 18, 1157-1161.

Goodacre, R., Vaidyanathan, S., Bianchi, G., \& Kell, D. B. (2002). Metabolic profiling using direct infusion electrospray ionisation mass spectrometry for the characterisation of olive oils. The Analyst, 11, 1457-1462.

Greenwood, D. (2000). Antimicrobial chemotherapy (4th ed.). Norfolk: Oxford University Press Inc.

Griffin, J. L., \& Kauppinen, R. A. (2007). Tumour metabolomics in animal models of human cancer. Journal of Proteome Research, 6, 498-505.

Han, X., \& Gross, R. W. (2005). Shotgun lipidomics: Electrospray ionization mass spectrometric analysis and quantitation of cellular lipidomes directly from crude extracts of biological samples. Mass Spectrometry Reviews, 24, 367-412.

Herrgård, M. J., Swainston, N., Dobson, P., Dunn, W. B., Arga, K. Y., Arvas, M., et al. (2008). A consensus yeast metabolic network reconstruction obtained from a community approach to systems biology. Nature Biotechnology, 26, 1155-1160.

Kaper, J. B., Nataro, J. B., \& Mobley, H. L. T. (2004). Pathogenic Escherichia coli. Nature Reviews Microbiology, 2, 123-140.

Kaplan, F., Kopka, J., Haskell, D. W., Zhao, W., Schiller, K. C., Gatzke, N., et al. (2004). Exploring the temperature-stress metabolome of Arabidopsis. Plant Physiology, 136, 4159-4168.

Kenny, L. C., Broadhurst, D. I., Dunn, W., Brown, M., North, R. A., McCowan, L., et al. (2010). Robust early pregnancy prediction of later preeclampsia using metabolomic biomarkers. Hypertension, 56, 741-749.

Koek, M. M., Muilwijk, B., van der Werf, M. J., \& Hankemeier, T. (2006). Microbial metabolomics with gas chromatography/mass spectrometry. Analytical Chemistry, 78, 1272-1281.

Kolak, M., Westerbacka, J., Velagapudi, V. R., Wagsater, D., Yetukuri, L., Makkonen, J., et al. (2007). Adipose tissue inflammation and increased ceramide content characterize subjects with high liver fat content independent of obesity. Diabetes, 56, 1960-1968.

Lau, S. H., Reddy, S., Cheesbrough, J., Bolton, F. J., Willshaw, G., Cheasty, T., et al. (2008). Major uropathogenic Escherichia coli strain isolated in the northwest of England identified by multilocus sequence typing. Journal of Clinical Microbiology, 46, 1076-1080.

Leying, H., Suerbaum, S., Kroll, H.-P., Karch, H., \& Opferkuch, W. (1986). Influence of B-lactam antibiotics and ciprofloxacin on composition and immunogenicity of Escherichia coli outer membrane. Antimicrobial Agents and Chemotherapy, 30, $475-480$

Lisec, J., Schauer, N., Kopka, J., Willmitzer, L., \& Fernie, A. R. (2006). Gas chromatography mass spectrometry-based metabolite profiling in plants. Nature Protocols, 1, 387-396.

Lowe, R. G. T., Allwood, J. W., Galster, A. M., Urban, M., Daudi, A., Canning, G., et al. (2010). A combined $1 \mathrm{H}$ nuclear magnetic resonance and electrospray ionization-mass spectrometry analysis to understand the basal metabolism of plant-pathogenic Fusarium spp. Molecular Plant-Microbe Interactions, 23, $1605-1618$.

MacKenzie, D. A., Defernez, M., Dunn, W. B., Brown, M., Fuller, L. J., Seco de Herrera, S. R. M., et al. (2008). Relatedness of medically important strains of Saccharomyces cerevisiae as revealed by phylogenetics and metabolomics. Yeast, 25, 501-512.

Mattila, I., Seppänen-Laakso, T., Suortti, T., \& Orešič, M. (2008). Application of lipidomics and metabolomics to the study of adipose tissue. Methods in Molecular Biology, 456, 123-130.

Merino, S., Doménech, O., Diez, I., Sanz, F., Vinas, M., Montero, M. T., et al. (2003). Effects of ciprofloxacin on Escherichia coli lipid bilayers: An Atomic Force Microscopy Study. Langmuir, 19, 6922-6927.

Mori, H. (2004). From the Sequence to Cell Modelling: Comprehensive Functional Genomics in Escherichia coli. Journal of Biochemistry and Molecular Biology, 37, 83-92.

Okusu, H., Ma, D., \& Nikaido, H. (1996). AcrAB efflux pump plays a major role in the antibiotic resistance phenotype of Escherichia coli multiple-antibiotic-resistance (Mar) mutants. Journal of Bacteriology, 178, 306-308.

Orešič, M., Simmel, S., Sysi-Aho, M., Näntö-Salonen, K., SeppänenLaakso, T., Parikka, V., et al. (2008). Dysregulation of lipid and amino acid metabolism precedes islet autoimmunity in children who later progress to type 1 diabetes. The Journal of Experimental Medicine, 205, 2975-2984.

Poole, K., Krebes, K., McNally, C., \& Neshat, S. (1993). Multiple antibiotic resistance in Pseudomonas aeruginosa: evidence for involvement of an efflux operon. Journal of Bacteriology, 22, 7363-7372.

Preisner, O., Almeida Lopes, A., Guiomar, R., Machado, J., \& Menezes, J. C. (2007). Fourier transform infrared (FT-IR) spectroscopy in bacteriology: towards a reference method for bacteria discrimination. Analytical and Bioanalytical Chemistry, 387, 1739-1748.

RajBhandary, U. L., \& Söll, D. (2008). Aminoacyl-tRNAs, the bacterial cell envelope, and antibiotics. Proceedings of the National Academy of Sciences, 105, 5285-5286.

Riley, M., Abe, T., Arnaud, M. B., Berlyn, M. K. B., Blattner, F. R., Chaudhuri, R. R., et al. (2006). Escherichia coli K-12: a cooperatively developed annotation snapshot-2005. Nucleic Acids Research, 34, 1-9.

Roessner, U., Luedemann, A., Brust, D., Fiehn, O., Linke, T., Willmitzer, L., et al. (2001). Metabolic profiling allows comprehensive phenotyping of genetically or environmentally modified plant systems. Plant Cell, 13, 11-29.

Rojas-Cherto, M., Peironcely, J. E., Kasper, P. T., van der Hooft, J. J. J., de Vos, R. C. H., Vreeken, R., et al. (2012). Metabolite identification using automated comparison of high-resolution multistage mass spectral trees. Analytical Chemistry, 84, 5524-5534.

Roux, A., Xu, Y., Heilier, J.-F., Olivier, M.-F., Ezan, E., Tabet, J.-C., et al. (2012). Annotation of the human adult urinary metabolome and metabolite identification using ultra high performance liquid chromatography coupled to a linear quadrupole ion trap-orbitrap mass spectrometer. Analytical Chemistry, 84, 6429-6437. 
Roy, H., \& Ibba, M. (2008). RNA-dependent lipid remodeling by bacterial multiple peptide resistance factors. Proceedings of the National Academy of Sciences, 105, 4667-4672.

Sáenz, Y., Briñas, L., Domínguez, E., Ruiz, J., Zarazaga, M., Vila, J., et al. (2004). Mechanisms of resistance in multiple-antibioticresistant Escherichia coli strains of human, animal, and food origins. Antimicrobial Agents and Chemotherapy, 48, 3996-4001.

Saito, K., \& Matsuda, F. (2010). Metabolomics for functional genomics, systems biology and biotechnology. Annual Review of Plant Biology, 61, 463-489.

Sreekumar, A., Poisson, L. M., Rajendiran, T. M., Khan, A. P., Cao, Q., Yu, J., et al. (2009). Metabolomic profiles delineate potential role for sarcosine in prostate cancer progression. Nature, 457, 910-914.

Sumner, L. W., Amberg, A., Barrett, D., Beale, M. H., Beger, R., Daykin, C. A., et al. (2007). Proposed minimum reporting standards for chemical analysis. Metabolomics, 3, 211-221.

Tikunov, Y., De Vos, C. H. R., Gonzalez-Paramas, A. M., Hall, R. D., \& Bovy, A. G. (2010). A role for differtential glycoconjugation in the emission of phenylpropanoid volatiles from tomato fruit discovered using a metabolic data fusion approach. Plant Physiology, 152, 55-70.

Tomasz, A. (1994). Multiple-antibiotic-resistant bacteria-A report on the Rockefeller University Workshop. The New England Journal of Medicine, 330, 1247-1251.

van der Hooft, J. J. J., Vervoort, J., Bino, R. J., Beekwilder, J., \& de Vos, R. C. H. (2011). Polyphenol identification based on systematic and robust high-resolution accurate mass spectrometry fragmentation. Analytical Chemistry, 83, 409-416.

van der Hooft, J. J. J., Vervoort, J., Bino, R. J., \& de Vos, R. C. H. (2012). Spectral trees as a robust annotation tool in LC-MS based metabolomics. Metabolomics, 8, 691-703.
Van Der Werf, M. J., Overkamp, K. M., Mulwijk, B., Koek, M. M., Van Der Werff-Van Der Vat, B. J. C., Jellema, R. H., Coulier, L. \& Hankemeier, T. (2008). Comprehensive analysis of the metabolome of Pseudomonas putida $\mathrm{S} 12$ grown on different carbon sources. Molecular Biosystems, 4, 315-327.

Velagapudi, V. R., Hezaveh, R., Reigstad, C. S., Gopalacharyulu, P., Yetukuri, L., Islam, S., et al. (2010). The gut microbiota modulates host energy and lipid metabolism in mice. Journal of Lipid Research, 51, 1101-1112.

Wedge, D. C., Allwood, J. W., Dunn, W. B., Vaughan, A. A., Simpson, K., Brown, M., et al. (2011). Is serum or plasma more appropriate for inter-subject comparisons in metabolomic studies? An assessment in patients with small-cell lung cancer. Analytical Chemistry, 83, 6689-6697.

Wehlri, P. M., Lindberg, E., Sparén, A., Josefson, M., Dunstan, R. H., Wold, A. E., et al. (2013). Exploring bacterial phenotypic diversity using factorial design and FTIR multivariate fingerprinting. Journal of Chemometrics. doi:10.1002/cem.2588.

Wiener, J., Quinn, J. P., Bradford, P. A., Goering, R. V., Nathan, C., Bush, K., et al. (1999). Multiple antibiotic-resistant Klebsiella and Escherichia coli in nursing homes. The Journal of the American Medical Association, 281, 517-523.

Winder, C. L., Dunn, W. B., Schuler, S., Broadhurst, D., Jarvis, R. M., Stephens, G. M., et al. (2008). Global metabolic profiling of Escherichia coli cultures: an evaluation of methods for quenching and extraction of intracellular metabolites. Analytical Chemistry, 80, 2939-2948.

Winder, C. L., Gordon, S. V., Dale, J., Hewinson, R. G., \& Goodacre, R. (2006). Metabolic fingerprints of Mycobacterium bovis cluster with molecular type: Implications for genotype-phenotype links. Microbiology, 152, 2757-2765. 